Article

\title{
Studies in Sums of Finite Products of the Second, Third, and Fourth Kind Chebyshev Polynomials
}

\author{
Taekyun Kim ${ }^{1,2, *}$, Dae San Kim ${ }^{3, * \mathbb{D}}$, Hyunseok Lee ${ }^{2}$ and Jongkyum Kwon ${ }^{4, *}$ \\ 1 School of Science, Xi'an Technological University, Xi'an 710021, China \\ 2 Department of Mathematics, Kwangwoon University, Seoul 139-701, Korea; luciasconstant@gmail.com \\ 3 Department of Mathematics, Sogang University, Seoul 121-742, Korea \\ 4 Department of Mathematics Education and ERI, Gyeongsang National University, Jinju 52828, Korea \\ * Correspondence: tkkim@kw.ac.kr (T.K.); dskim@sogang.ac.kr (D.S.K.); mathkjk26@gnu.ac.kr (J.K.)
}

Received: 2 January 2020; Accepted: 4 February 2020; Published: 7 February 2020

\begin{abstract}
In this paper, we consider three sums of finite products of Chebyshev polynomials of two different kinds, namely sums of finite products of the second and third kind Chebyshev polynomials, those of the second and fourth kind Chebyshev polynomials, and those of the third and fourth kind Chebyshev polynomials. As a generalization of the classical linearization problem, we represent each of such sums of finite products as linear combinations of Hermite, generalized Laguerre, Legendre, Gegenbauer, and Jacobi polynomials. These are done by explicit computations and the coefficients involve terminating hypergeometric functions ${ }_{2} F_{1,1} F_{1},{ }_{2} F_{2}$, and ${ }_{4} F_{3}$.
\end{abstract}

Keywords: sums of finite products; Chebyshev polynomials of the second; third and fourth kinds; terminating hypergeometric functions

MSC: 11B83; 33C05; 33C15; 33C20; 33C45

\section{Introduction and Preliminaries}

The classical linearization problem consists of determining the coefficients $c_{n, m}(k)$ in the expansion of the product of two polynomials $q_{n}(x)$ and $r_{m}(x)$ in terms of arbitrary polynomial sequence $\left\{p_{k}(x)\right\}_{k \geq 0}$. See [1].

$$
q_{n}(x) r_{m}(x)=\sum_{k=0}^{n+m} c_{n m}(k) p_{k}(x) .
$$

There are several special cases of this: If $q_{n}(x)=r_{n}(x)=p_{n}(x)$, then it is called either the standard linearization or a Clebsch-Gordan-type problem:

$$
q_{n}(x) q_{m}(x)=\sum_{k=0}^{n+m} c_{n m}(k) q_{k}(x)
$$

If $r_{m}(x)=1$, then it is known as the connection problem:

$$
q_{n}(x)=\sum_{k=0}^{n} c_{n}(k) p_{k}(x)
$$

If, furthermore, $q_{n}(x)=x^{n}$ in (3), then it is called the inversion problem. 
Let $n, r, s$ be nonnegative integers with $r+s \geq 1$. Here, as one motivation for the present research, we would like to generalize the linearization problem in (1) and consider the following three sums of finite products of Chebyshev polynomials of two different kinds:

$$
\begin{aligned}
& \alpha_{n, r, s}(x)=\sum_{i_{1}+\cdots+i_{r}+j_{1}+\cdots+j_{s}=n} U_{i_{1}}(x) \cdots U_{i_{r}}(x) V_{j_{1}}(x) \cdots V_{j_{s}}(x), \\
& \beta_{n, r, s}(x)=\sum_{i_{1}+\cdots+i_{r}+j_{1}+\cdots+j_{s}=n} U_{i_{1}}(x) \cdots U_{i_{r}}(x) W_{j_{1}}(x) \cdots W_{j_{s}}(x), \\
& \gamma_{n, r, s}(x)=\sum_{i_{1}+\cdots+i_{r}+j_{1}+\cdots+j_{s}=n} V_{i_{1}}(x) \cdots V_{i_{r}}(x) W_{j_{1}}(x) \cdots W_{j_{s}}(x),
\end{aligned}
$$

where $U_{n}(x), V_{n}(x)$, and $W_{n}(x)$ are respectively Chebyshev polynomials of the second, third, and fourth kinds, and the sums are over all nonnegative integers, $i_{1}, \ldots, i_{r}, j_{1}, \ldots, j_{s}$ with $i_{1}+\cdots+i_{r}+$ $j_{1}+\cdots+j_{s}=n$. Then, we will represent each of the sums of finite products in Equations (4)-(6) as linear combinations of Hermite polynomials $H_{n}(x)$, generalized Laguerre polynomials $L_{n}^{\alpha}(x)$, Legendre polynomials $P_{n}(x)$, Gegenbauer polynomials $C_{n}^{(\lambda)}(x)$, and Jacobi polynomials $P_{n}^{(\alpha, \beta)}(x)$ (see Theorems 1-3).

As another motivation for the present study, we would like to mention a convolution identity of Bernoulli polynomials that yields the famous Faber-Pandharipande-Zagier identity and Miki's identity. For this, let us first recall that the Bernoulli polynomials are given by

$$
\frac{t}{e^{t}-1} e^{x t}=\sum_{n=0}^{\infty} B_{n}(x) \frac{t^{n}}{n !}
$$

Then, let us put

$$
\mathcal{E}_{m}(x)=\sum_{k=1}^{m-1} \frac{1}{k(m-k)} B_{k}(x) B_{m-k}(x), \quad(m \geq 2) .
$$

In the Introduction of [2], we noted that the following identity can be derived from the Fourier expansion of $\mathcal{E}_{m}(\langle x\rangle)$. Here, $\langle x\rangle=x-[x]$ is the fractional part of $x$, for any real number $x$ :

$$
\sum_{k=1}^{m-1} \frac{1}{k(m-k)} B_{k}(x) B_{m-k}(x)=\frac{2}{m^{2}}\left(B_{m}+\frac{1}{2}\right)+\frac{2}{m} \sum_{k=1}^{m-2} \frac{\left(\begin{array}{c}
m \\
m
\end{array}\right)}{m-k} B_{m-k} B_{k}(x)+\frac{2}{m} H_{m-1} B_{m}(x),(m \geq 2),
$$

where $H_{m}=\sum_{j=1}^{m} \frac{1}{j}$ are the harmonic numbers.

Furthermore, (7) can be modified to give

$$
\begin{aligned}
& \sum_{k=1}^{m-1} \frac{1}{2 k(2 m-2 k)} B_{2 k}(x) B_{2 m-2 k}(x)+\frac{2}{2 m-1} B_{1}(x) B_{2 m-1}(x) \\
& =\frac{1}{m} \sum_{k=1}^{m} \frac{1}{2 k}\left(\begin{array}{c}
2 m \\
2 k
\end{array}\right) B_{2 k} B_{2 m-2 k}(x)+\frac{1}{m} H_{2 m-1} B_{2 m}(x)+\frac{2}{2 m-1} B_{2 m-1} B_{1}(x), \quad(m \geq 2) .
\end{aligned}
$$

Let $x=\frac{1}{2}$ and $x=0$ in (8) give respectively Faber-Pandharipande-Zagier identity (see [3]) and a slight variant of Miki's identity (see [4-7]). It is worth noting that our methods are very simple at the level of Fourier series expansions, whereas the other approaches in [4-7] use different methods from one another and are quite involved.

Before we state our main results in Theorems 1-3, we will fix notations that will be used throughout this paper and recall some basic facts about orthogonal polynomials that will be needed. 
Let $n$ be a nonnegative integer. Then, the falling factorial polynomials $(x)_{n}$ and the rising factorial polynomials $\langle x\rangle_{n}$ are respectively given by

$$
\begin{aligned}
& (x)_{0}=1, \quad(x)_{n}=x(x-1) \cdots(x-n+1), \quad(n \geq 1), \\
& \langle x\rangle_{0}=1, \quad\langle x\rangle_{n}=x(x+1) \cdots(x+n-1), \quad(n \geq 1) .
\end{aligned}
$$

The two factorial polynomials are related by

$$
\begin{gathered}
(-1)^{n}(x)_{n}=\langle-x\rangle_{n}, \quad(-1)^{n}\langle x\rangle_{n}=(-x)_{n} . \\
\frac{(2 n-2 j) !}{(n-j) !}=\frac{2^{2 n-2 j}(-1)^{j}\left\langle\frac{1}{2}\right\rangle_{n}}{\left\langle\frac{1}{2}-n\right\rangle_{j}}, \quad(n \geq j \geq 0) . \\
\frac{(2 n+2 j) !}{(n+j) !}=2^{2 n+2 j}\left\langle\frac{1}{2}\right\rangle_{n}\left\langle n+\frac{1}{2}\right\rangle_{j}, \quad(n, j \geq 0) . \\
\Gamma\left(n+\frac{1}{2}\right)=\frac{(2 n) ! \sqrt{\pi}}{2^{2 n} n !}, \quad(n \geq 0), \\
\frac{\Gamma(x+1)}{\Gamma(x+1-n)}=(x)_{n}, \quad \frac{\Gamma(x+n)}{\Gamma(x)}=\langle x\rangle_{n}, \quad(n \geq 0),
\end{gathered}
$$

where $\Gamma(x)$ is the gamma function. The hypergeometric function is defined by

$$
{ }_{p} F_{q}\left(a_{1}, \ldots, a_{p} ; b_{1}, \ldots, b_{q} ; x\right)=\sum_{n=0}^{\infty} \frac{\left\langle a_{1}\right\rangle_{n} \cdots\left\langle a_{p}\right\rangle_{n}}{\left\langle b_{1}\right\rangle_{n} \cdots\left\langle b_{q}\right\rangle_{n}} \frac{x^{n}}{n !} .
$$

Next, we will recall some very basic facts about Chebyshev polynomials of the second kind $U_{n}(x)$, the third kind $V_{n}(x)$, and the fourth kind $W_{n}(x)$ (see [8,9]). In addition, we will state those facts about Hermite polynomials $H_{n}(x)$, extended Laguerre polynomials $L_{n}^{\alpha}(x)$, Legendre polynomials $P_{n}(x)$, Gegenbauer polynomials $C_{n}^{(\lambda)}(x)$, and Jacobi polynomials $P_{n}^{(\alpha, \beta)}(x)$ (see [10-14]). We let the reader refer to the standard books [15-17] for further details on these family of orthogonal polynomials.

In terms of generating functions, the above mentioned orthogonal polynomials are given as follows:

$$
\begin{gathered}
\frac{1}{1-2 x t+t^{2}}=\sum_{n=0}^{\infty} U_{n}(x) t^{n}, \\
\frac{1-t}{1-2 x t+t^{2}}=\sum_{n=0}^{\infty} V_{n}(x) t^{n} \\
\frac{1+t}{1-2 x t+t^{2}}=\sum_{n=0}^{\infty} W_{n}(x) t^{n}, \\
e^{2 x t-t^{2}}=\sum_{n=0}^{\infty} H_{n}(x) \frac{t^{n}}{n !}, \\
(1-t)^{-\alpha-1} \exp \left(-\frac{x t}{1-t}\right)=\sum_{n=0}^{\infty} L_{n}^{\alpha}(x) t^{n}, \quad(\alpha>-1), \\
\left(1-2 x t+t^{2}\right)^{-\frac{1}{2}}=\sum_{n=0}^{\infty} P_{n}(x) t^{n}, \quad \\
\frac{1}{\left(1-2 x t+t^{2}\right)^{\lambda}}=\sum_{n=0}^{\infty} C_{n}^{(\lambda)}(x) t^{n}, \quad\left(\lambda>-\frac{1}{2}, \lambda \neq 0,|t|<1,|x| \leq 1\right),
\end{gathered}
$$




$$
\frac{\alpha+\beta}{R(1-t+R)^{\alpha}(1+t+R)^{\beta}}=\sum_{n=0}^{\infty} P_{n}^{(\alpha, \beta)}(x) t^{n}, \quad\left(R=\sqrt{1-2 x t+t^{2}}, \alpha, \beta>-1\right) .
$$

In terms of explicit expressions, they are given as follows:

$$
\begin{aligned}
& U_{n}(x)=(n+1)_{2} F_{1}\left(-n, n+2 ; \frac{3}{2} ; \frac{1-x}{2}\right)=\sum_{l=0}^{\left[\frac{n}{2}\right]}(-1)^{l}\left(\begin{array}{c}
n-l \\
l
\end{array}\right)(2 x)^{n-2 l}, \quad(n \geq 0) \\
& V_{n}(x)={ }_{2} F_{1}\left(-n, n+1 ; \frac{1}{2} ; \frac{1-x}{2}\right)=\sum_{l=0}^{n}\left(\begin{array}{c}
n+l \\
2 l
\end{array}\right) 2^{l}(x-1)^{l}, \quad(n \geq 0), \\
& W_{n}(x)=(2 n+1){ }_{2} F_{1}\left(-n, n+1 ; \frac{3}{2} ; \frac{1-x}{2}\right) \\
& =(2 n+1) \sum_{l=0}^{n} \frac{2^{l}}{2 l+1}\left(\begin{array}{c}
n+l \\
2 l
\end{array}\right)(x-1)^{l}, \quad(n \geq 0), \\
& H_{n}(x)=n ! \sum_{l=0}^{\left[\frac{n}{2}\right]} \frac{(-1)^{l}}{l !(n-2 l) !}(2 x)^{n-2 l}, \quad(n \geq 0), \\
& L_{n}^{\alpha}(x)=\frac{\langle\alpha+1\rangle_{n}}{n !} F_{1}(-n ; \alpha+1 ; x)=\sum_{l=0}^{n} \frac{(-1)^{l}\left(\begin{array}{c}
n+\alpha \\
n-l
\end{array}\right)}{l !} x^{l}, \quad(n \geq 0), \\
& P_{n}(x)={ }_{2} F_{1}\left(-n, n+1 ; 1 ; \frac{1-x}{2}\right)=\frac{1}{2^{n}} \sum_{l=0}^{\left[\frac{n}{2}\right]}(-1)^{l}\left(\begin{array}{c}
n \\
l
\end{array}\right)\left(\begin{array}{c}
2 n-2 l \\
n
\end{array}\right) x^{n-2 l},(n \geq 0), \\
& C_{n}^{(\lambda)}(x)=\left(\begin{array}{c}
n+2 \lambda-1 \\
n
\end{array}\right){ }_{2} F_{1}\left(-n, n+2 \lambda ; \lambda+\frac{1}{2} ; \frac{1-x}{2}\right) \\
& =\sum_{k=0}^{\left[\frac{n}{2}\right]}(-1)^{k} \frac{\Gamma(n-k+\lambda)}{\Gamma(\lambda) k !(n-2 k) !}(2 x)^{n-2 k},(n \geq 0), \\
& P_{n}^{(\alpha, \beta)}(x)=\frac{\langle\alpha+1\rangle_{n}}{n !}{ }_{2} F_{1}\left(-n, 1+\alpha+\beta+n ; \alpha+1 ; \frac{1-x}{2}\right) \\
& =\sum_{k=0}^{n}\left(\begin{array}{c}
n+\alpha \\
n-k
\end{array}\right)\left(\begin{array}{c}
n+\beta \\
k
\end{array}\right)\left(\frac{x-1}{2}\right)^{k}\left(\frac{x+1}{2}\right)^{n-k}, \quad(n \geq 0) \text {. }
\end{aligned}
$$

\section{Statements of Results}

The following three theorems are the main results of this paper, all of which are new. Here, we note that we treat sums of finite products of Chebyshev polynomials of two different kinds, whereas all the results so far, except for [18], treated sums of finite products of some polynomials of single kind.

Theorem 1. Let $n, r$, s be nonnegative integers with $r+s \geq 1$. Then, we have the following identities:

$$
\sum_{i_{1}+\cdots+i_{r}+j_{1}+\cdots+j_{s}=n} U_{i_{1}}(x) \cdots U_{i_{r}}(x) V_{j_{1}}(x) \cdots V_{j_{s}}(x)
$$




$$
\begin{aligned}
& =\sum_{k=0}^{n} \frac{(-1)^{n-k}}{(r+s-1) ! k !} \sum_{j=0}^{\left[\frac{n-k}{2}\right]} \frac{\left(\begin{array}{c}
s \\
n-k-2 j
\end{array}\right)(r+s+k+2 j-1) !}{j !} \\
& \times{ }_{1} F_{1}(-j ; 1-r-s-k-2 j ;-1) H_{k}(x) \\
& =\sum_{k=0}^{n} \frac{2^{n}(-1)^{n-k}}{(r+s-1) ! \Gamma(\alpha+n-k+1)} \\
& \times \sum_{m=0}^{\left[\frac{k}{2}\right]} \frac{\left(-\frac{1}{4}\right)^{m}(n+r+s-m-1) ! \Gamma(n+\alpha-2 m+1)}{m !(k-2 m) !{ }_{2} F_{2}\left(2 m-k,-s ; m+1-n-r-s, 2 m-n-\alpha ;-\frac{1}{2}\right)} L_{n-k}^{\alpha}(x) \\
& =\sum_{k=0}^{n} \frac{(-1)^{n-k} 2^{k}(2 k+1)}{(r+s-1) !} \sum_{j=0}^{\left[\frac{n-k}{2}\right]} \frac{2^{j}\left(\begin{array}{c}
s \\
n-k-2 j
\end{array}\right)(r+s+k+2 j-1) !}{j !(2 k+2 j+1) ! !} \\
& \times{ }_{2} F_{1}\left(-j,-k-j-\frac{1}{2} ; 1-r-s-k-2 j ; 1\right) P_{k}(x) \\
& =\sum_{k=0}^{n} \frac{(-1)^{n-k} \Gamma(\lambda)(k+\lambda)}{(r+s-1) !} \sum_{j=0}^{\left[\frac{n-k}{2}\right]} \frac{\left(\begin{array}{c}
s \\
n-k-2 j
\end{array}\right)(r+s+k+2 j-1) !}{j ! \Gamma(k+\lambda+j+1)} \\
& \times{ }_{2} F_{1}(-j,-k-\lambda-j ; 1-r-s-k-2 j ; 1) C_{k}^{(\lambda)}(x) \\
& =\sum_{k=0}^{n} \frac{(-2)^{n+k} \Gamma(k+\alpha+\beta+1)}{(r+s-1) ! \Gamma(2 k+\alpha+\beta+1)} \sum_{m=0}^{\left[\frac{n-k}{2}\right]} \frac{\left(-\frac{1}{4}\right)^{m}}{m !} \\
& \times \sum_{l=0}^{n-k-2 m} \frac{\left(\frac{1}{2}\right)^{l}\left(\begin{array}{l}
s \\
l
\end{array}\right)(n+r+s-l-m-1) !}{(n-k-l-2 m) !} \\
& \times{ }_{2} F_{1}(k+l+2 m-n, k+\beta+1 ; 2 k+\alpha+\beta+2 ; 2) P_{k}^{(\alpha, \beta)}(x) \text {. }
\end{aligned}
$$

Here, $(2 n-1) ! !=(2 n-1)(2 n-3) \cdots 1$, for $n \geq 1$, and $(-1) ! !=1$.

Theorem 2. Let $n, r, s$ be nonnegative integers with $r+s \geq 1$. Then, we have the following representations:

$$
\begin{gathered}
\sum_{i_{1}+\cdots+i_{r}+j_{1}+\cdots+j_{s}=n} U_{i_{1}}(x) \cdots U_{i_{r}}(x) W_{j_{1}}(x) \cdots W_{j_{s}}(x) \\
=\sum_{k=0}^{n} \frac{1}{(r+s-1) ! k !} \sum_{j=0}^{\left[\frac{n-k}{2}\right]} \frac{\left(\begin{array}{c}
s \\
n-k-2 j
\end{array}\right)(r+s+k+2 j-1) !}{j !} \\
\quad \times{ }_{1} F_{1}(-j ; 1-r-s-k-2 j ;-1) H_{k}(x) \\
=\sum_{k=0}^{n} \frac{2^{n}(-1)^{n-k}}{(r+s-1) ! \Gamma(\alpha+n-k+1)} \sum_{m=0}^{\left[\frac{k}{2}\right]} \frac{\left(-\frac{1}{4}\right)^{m}(n+r+s-m-1) ! \Gamma(n+\alpha-2 m+1)}{m !(k-2 m) !} \\
\quad \times{ }_{2} F_{2}\left(2 m-k,-s ; m+1-n-r-s, 2 m-n-\alpha ; \frac{1}{2}\right) L_{n-k}^{\alpha}(x)
\end{gathered}
$$




$$
\begin{aligned}
= & \sum_{k=0}^{n} \frac{(2 k+1) 2^{k}}{(r+s-1) !} \sum_{j=0}^{\left[\frac{n-k}{2}\right]} \frac{2^{j}\left(\begin{array}{c}
s \\
n-k-2 j
\end{array}\right)(r+s+k+2 j-1) !}{j !(2 k+2 j+1) ! !} \\
& \times{ }_{2} F_{1}\left(-j,-k-j-\frac{1}{2} ; 1-r-s-k-2 j ; 1\right) P_{k}(x) \\
= & \sum_{k=0}^{n} \frac{\Gamma(\lambda)(k+\lambda)}{(r+s-1) !} \sum_{j=0}^{\left[\frac{n-k}{2}\right]} \frac{\left(\begin{array}{c}
s \\
n-k-2 j
\end{array}\right)(r+s+k+2 j-1) !}{j ! \Gamma(k+\lambda+j+1)} \\
= & \times{ }_{2} F_{1}(-j,-k-\lambda-j ; 1-r-s-k-2 j ; 1) C_{k}^{(\lambda)}(x) \\
& \quad \frac{(-2)^{n+k} \Gamma(k+\alpha+\beta+1)}{(r+s-1) ! \Gamma(2 k+\alpha+\beta+1)} \sum_{m=0}^{n} \frac{\left(-\frac{n-k}{2}\right)^{m}}{m !} \\
& \times{ }_{2}^{n-k-2 m} \frac{\left(-\frac{1}{2}\right)^{l}\left(\begin{array}{l}
s \\
l
\end{array}\right)(n+r+s-l-m-1) !}{(n-k-l-2 m) !}
\end{aligned}
$$

Theorem 3. Let $n, r, s$ be nonnegative integers with $r+s \geq 1$. Then, we have the following expressions:

$$
\begin{aligned}
& \sum_{i_{1}+\cdots+i_{r}+j_{1}+\cdots+j_{s}=n} V_{i_{1}}(x) \cdots V_{i_{r}}(x) W_{j_{1}}(x) \cdots W_{j_{s}}(x) \\
&=\sum_{k=0}^{n} \frac{1}{(r+s-1) ! k !} \sum_{l=0}^{\left[\frac{n-k}{2}\right]} \frac{(r+s+k+2 l-1) !\left(\begin{array}{c}
s \\
n-k-2 l
\end{array}\right)}{l !} \\
& \quad \times{ }_{1} F_{1}(-l ; 1-r-s-k-2 l ;-1) \\
& \quad \times{ }_{2} F_{1}(k+2 l-n,-r ; s+k+2 l-n+1 ;-1) H_{k}(x) \\
&=\sum_{k=0}^{n} \frac{2^{n}(-1)^{k}}{(r+s-1) ! \Gamma(\alpha+k+1)} \\
& \quad \times \sum_{i=0}^{n-k} 2^{-i}\left(\begin{array}{l}
s \\
i
\end{array}\right){ }_{2} F_{1}(-i,-r ; s-i+1 ;-1) \\
& \quad \times \frac{\left[\frac{n-k-i}{2}\right]\left(-\frac{1}{4}\right)^{m}(n+r+s-i-m-1) ! \Gamma(n+\alpha-i-2 m+1)}{m !(n-k-i-2 m) !} L_{k}^{\alpha}(x) \\
&=\sum_{k=0}^{n} \frac{(2 k+1) 2^{k}\left[\frac{n-k}{2}\right]}{(r+s-1) !} \sum_{l=0}^{l} \frac{2^{l}(r+s+k+2 l-1) !\left(\begin{array}{c}
s \\
n-k-2 l
\end{array}\right)}{(2 k+2 l+1) ! ! l !} \\
& \quad \times{ }_{2} F_{1}\left(-l,-k-l-\frac{1}{2} ; 1-r-s-k-2 l ; 1\right) \\
& \quad \times{ }_{2} F_{1}(k+2 l-n,-r ; s+k+2 l-n+1 ;-1) P_{k}(x) \\
&=\sum_{k=0}^{n} \frac{(k+\lambda) \Gamma(\lambda)}{(r+s-1) !} \sum_{l=0}^{\left[\frac{n-k}{2}\right]} \frac{(r+s+k+2 l-1) !\left(\begin{array}{c}
s \\
n-k-2 l
\end{array}\right)}{l ! \Gamma(k+l+\lambda+1)} \\
& \quad \times{ }_{2} F_{1}(-l,-k-l-\lambda ; 1-r-s-k-2 l ; 1) \\
& \quad \times{ }_{2} F_{1}(k+2 l-n,-r ; s+k+2 l-n+1 ;-1) C_{k}^{(\lambda)}(x)
\end{aligned}
$$




$$
\begin{aligned}
= & \sum_{k=0}^{n} \frac{(-2)^{n+k} \Gamma(k+\alpha+\beta+1)}{(r+s-1) ! \Gamma(2 k+\alpha+\beta+1)} \sum_{i=0}^{n-k}\left(-\frac{1}{2}\right)^{i}\left(\begin{array}{l}
s \\
i
\end{array}\right){ }_{2} F_{1}(-i,-r ; s-i+1 ;-1) \\
& \times \sum_{m=0}^{\left[\frac{n-k-i}{2}\right]} \frac{\left(-\frac{1}{4}\right)^{m}(n+r+s-i-m-1) !}{m !(n-k-i-2 m) !} \\
& \times{ }_{2} F_{1}(k+i+2 m-n, k+\beta+1 ; 2 k+\alpha+\beta+2 ; 2) P_{k}^{(\alpha, \beta)}(x) .
\end{aligned}
$$

Before we move on to the next section, we would like to recall some of the related previous works. In [19], sums of finite products of Chebyshev polynomials of the first, third, and and fourth kinds were represented in terms of $H_{n}(x), L_{n}^{\alpha}(x), P_{n}(x), C_{n}^{(\lambda)}(x), P_{n}^{(\alpha, \beta)}(x)$. In addition, in [20], sums of finite products of Chebyshev polynomials of the second kind were expressed in terms of the same orthogonal polynomials. Here, we emphasize that, except for the paper [18], which considered the sums of finite products in (4)-(6) and represented them in terms of all kinds of Chebyshev polynomials, all of the results so far have treated sums of finite products of some polynomials of single type. For further details on these, we let the reader refer to the references in $[19,20]$.

\section{Proofs of Theorems 1 and 2}

In this section, we will show Theorems 1 and 2. For this, we first state Propositions 1 and 2 that will be needed in showing Theorems 1-3. Here, we note that (a), (b), (c), (d), and (e) of Proposition 1 are respectively from (3.7) of [11], (2.3) of [13], (2.3) of [12], (2.3) of [9], and (2.7) of [14]. In fact, all the formulas in Proposition 1 follow from the orthogonalities of $H_{n}(x), L_{n}^{\alpha}(x), P_{n}(x), C_{n}^{(\lambda)}(x), P_{n}^{(\alpha, \beta)}(x)$ (see (32)-(36) in [19]), and Rodrigues' and Rodrigues-type formulas for those orthogonal polynomials (see (27)-(31)), and integration by parts.

Proposition 1. Let $q(x) \in \mathbb{R}[x]$ be a polynomial of degree $n$. Then, the following hold:

(a) $q(x)=\sum_{k=0}^{n} C_{k, 1} H_{k}(x)$, where

$$
C_{k, 1}=\frac{(-1)^{k}}{2^{k} k ! \sqrt{\pi}} \int_{-\infty}^{\infty} q(x) \frac{d^{k}}{d x^{k}} e^{-x^{2}} d x
$$

(b) $q(x)=\sum_{k=0}^{n} C_{k, 2} L_{k}^{\alpha}(x)$, where

$$
C_{k, 2}=\frac{1}{\Gamma(\alpha+k+1)} \int_{0}^{\infty} q(x) \frac{d^{k}}{d x^{k}}\left(e^{-x} x^{k+\alpha}\right) d x
$$

(c) $q(x)=\sum_{k=0}^{n} C_{k, 3} P_{k}(x)$, where

$$
C_{k, 3}=\frac{2 k+1}{2^{k+1} k !} \int_{-1}^{1} q(x) \frac{d^{k}}{d x^{k}}\left(x^{2}-1\right)^{k} d x .
$$

(d) $q(x)=\sum_{k=0}^{n} C_{k, 4} C_{k}^{(\lambda)}(x)$, where

$$
C_{k, 4}=\frac{(k+\lambda) \Gamma(\lambda)}{(-2)^{k} \sqrt{\pi} \Gamma\left(k+\lambda+\frac{1}{2}\right)} \int_{-1}^{1} q(x) \frac{d^{k}}{d x^{k}}\left(1-x^{2}\right)^{k+\lambda-\frac{1}{2}} d x .
$$


(e) $q(x)=\sum_{k=0}^{n} C_{k, 5} P_{k}^{(\alpha, \beta)}(x)$, where

$$
C_{k, 5}=\frac{(-1)^{k}(2 k+\alpha+\beta+1) \Gamma(k+\alpha+\beta+1)}{2^{\alpha+\beta+k+1} \Gamma(\alpha+k+1) \Gamma(\beta+k+1)} \int_{-1}^{1} q(x) \frac{d^{k}}{d x^{k}}(1-x)^{k+\alpha}(1+x)^{k+\beta} d x
$$

The next proposition was stated in [19].

Proposition 2. Let $m, k$ be nonnegative integers. Then, we have the following:

(a) $\int_{-\infty}^{\infty} x^{m} e^{-x^{2}} d x=\left\{\begin{array}{cl}0, & \text { if } m \equiv 1(\bmod 2), \\ \frac{m ! \sqrt{\pi}}{\left(\frac{m}{2}\right) ! 2^{m}}, & \text { if } m \equiv 0(\bmod 2) .\end{array}\right.$

(b) $\int_{-1}^{1} x^{m}\left(1-x^{2}\right)^{k} d x=\left\{\begin{array}{cl}0, & \text { if } m \equiv 1(\bmod 2), \\ \frac{2^{2 k+2} k ! m !\left(k+\frac{m}{2}+1\right) !}{\left(\frac{m}{2}\right) !(2 k+m+2) !}, & \text { if } m \equiv 0(\bmod 2) .\end{array}\right.$

(c) $\int_{-1}^{1} x^{m}\left(1-x^{2}\right)^{k+\lambda-\frac{1}{2}} d x=\left\{\begin{array}{cl}0, & \text { if } m \equiv 1(\bmod 2), \\ \frac{\Gamma\left(k+\lambda+\frac{1}{2}\right) \Gamma\left(\frac{m}{2}+\frac{1}{2}\right)}{\Gamma\left(k+\lambda+\frac{m}{2}+1\right)}, & \text { if } m \equiv 0(\bmod 2) .\end{array}\right.$

(d) $\int_{-1}^{1} x^{m}(1-x)^{k+\alpha}(1+x)^{k+\beta} d x=2^{2 k+\alpha+\beta+1} \sum_{s=0}^{m}\left(\begin{array}{c}m \\ s\end{array}\right)(-1)^{m-s} 2^{s} \frac{\Gamma(k+\alpha+1) \Gamma(k+\beta+s+1)}{\Gamma(2 k+\alpha+\beta+s+2)}$.

Lemmas 1 and 2 in the following can be shown by using (17)-(19) and were derived in [18]. However, for the sake of completeness and in view of its importance, we repeat the proof for Lemma 1. Lemma 2 can be proved analogously to Lemma 1.

Lemma 1. Let $n, r, s$ be nonnegative integers with $r+s \geq 1$. Then, we have the following identity:

$$
\sum_{i_{1}+\cdots+i_{r}+j_{1}+\cdots+j_{s}=n} U_{i_{1}}(x) \cdots U_{i_{r}}(x) V_{j_{1}}(x) \cdots V_{j_{s}}(x)=\frac{1}{(r+s-1) ! 2^{r+s-1}} \sum_{l=0}^{n}\left(\begin{array}{c}
s \\
l
\end{array}\right)(-1)^{l} U_{n+r+s-l-1}^{(r+s-1)}(x),
$$

where the sum is over all nonnegative integers $i_{1}, \ldots, i_{r}, j_{1}, \ldots, j_{s}$, with $i_{1}+\cdots+i_{r}+j_{1}+\cdots+j_{s}=n$.

Proof. Let $F(t, x)=\left(1-2 x t+t^{2}\right)^{-1}$. Then, we observe that

$$
\frac{\partial^{r+s-1}}{\partial x^{r+s-1}} F(t, x)=(r+s-1) !(2 t)^{r+s-1}\left(1-2 x t+t^{2}\right)^{-(r+s)} .
$$


Now, by making use of (17), (18), and (49), we have

$$
\begin{aligned}
& \sum_{n=0}^{\infty}\left(\sum_{i_{1}+\cdots i_{r}+j_{1}+\cdots+j_{s}=n} U_{i_{1}}(x) \cdots U_{i_{r}}(x) V_{j_{1}}(x) \cdots V_{j_{s}}(x)\right) t^{n} \\
& \quad=\left(\sum_{i=0}^{\infty} U_{i}(x) t^{i}\right)\left(\sum_{j=0}^{\infty} V_{j}(x) t^{j}\right)^{s} \\
& \quad=\left(\frac{1}{1-2 x t+t^{2}}\right)^{r}\left(\frac{1-t}{1-2 x t+t^{2}}\right)^{s} \\
& =(1-t)^{s}\left(1-2 x t+t^{2}\right)^{-(r+s)} \\
& =\frac{(1-t)^{s}}{(r+s-1) ! 2^{r+s-1}} \frac{1}{t^{r+s-1}} \frac{\partial^{r+s-1}}{\partial x^{r+s-1}} F(t, x) \\
& \quad=\frac{(1-t)^{s}}{(r+s-1) ! 2^{r+s-1}} \frac{1}{t^{r+s-1}} \sum_{m=0}^{\infty} U_{m+r+s-1}^{(r+s-1)}(x) t^{m+r+s-1} \\
& \quad=\frac{1}{(r+s-1) ! 2^{r+s-1}} \sum_{l=0}^{\infty}\left(\begin{array}{l}
s \\
l
\end{array}\right)(-1)^{l} t^{l} \sum_{m=0}^{\infty} U_{m+r+s-1}^{(r+s-1)}(x) t^{m} \\
&
\end{aligned}
$$

which completes the proof for (48).

Lemma 2. Let $n, r$, s be nonnegative integers with $r+s \geq 1$. Then, the following identity holds:

$$
\sum_{i_{1}+\cdots+i_{r}+j_{1}+\cdots+j_{s}=n} U_{i_{1}}(x) \cdots U_{i_{r}}(x) W_{j_{1}}(x) \cdots W_{j_{s}}(x)=\frac{1}{(r+s-1) ! 2^{r+s-1}} \sum_{l=0}^{n}\left(\begin{array}{c}
s \\
l
\end{array}\right) U_{n+r+s-l-1}^{(r+s-1)}(x),
$$

where the sum is over all nonnegative integers $i_{1}, \ldots, i_{r}, j_{1}, \ldots, j_{s}$, with $i_{1}+\cdots+i_{r}+j_{1}+\cdots+j_{s}=n$.

From (25), we see that the $r$ th derivative of $U_{n}(x)$ is given by

$$
U_{n}^{(r)}(x)=\sum_{m=0}^{\left[\frac{n-r}{2}\right]}(-1)^{m}\left(\begin{array}{c}
n-m \\
m
\end{array}\right)(n-2 m)_{r} 2^{n-2 m} x^{n-2 m-r},
$$

from which we have

$$
\begin{aligned}
& U_{n+r+s-l-1}^{(r+s+k-1)}(x)=\sum_{m=0}^{\left[\frac{n-k-l}{2}\right]}(-1)^{m}\left(\begin{array}{c}
n+r+s-l-m-1 \\
m
\end{array}\right) \\
& \times(n+r+s-l-2 m-1)_{r+s+k-1} 2^{n+r+s-l-2 m-1} x^{n-k-l-2 m} .
\end{aligned}
$$

Now, we are going to show Theorem 1 . With $\alpha_{n, r, s}(x)$ as in (4), we put

$$
\alpha_{n, r, s}(x)=\sum_{k=0}^{n} C_{k, 1} H_{k}(x)
$$


Then, from (a) of Proposition 1, (48), and (52), and integration by parts $k$ times, we have

$$
\begin{aligned}
C_{k, 1} & =\frac{(-1)^{k}}{2^{k} k ! \sqrt{\pi}} \int_{-\infty}^{\infty} \alpha_{n, r, s}(x) \frac{d^{k}}{d x^{k}} e^{-x^{2}} d x \\
& =\frac{(-1)^{k}}{2^{k} k ! \sqrt{\pi}(r+s-1) ! 2^{r+s-1}} \sum_{l=0}^{n}\left(\begin{array}{l}
s \\
l
\end{array}\right)(-1)^{l} \int_{-\infty}^{\infty} U_{n+r+s-l-1}^{(r+s-1)}(x) \frac{d^{k}}{d x^{k}} e^{-x^{2}} d x \\
& =\frac{(-1)^{k}}{2^{k} k ! \sqrt{\pi}(r+s-1) ! 2^{r+s-1}} \sum_{l=0}^{n-k}\left(\begin{array}{l}
s \\
l
\end{array}\right)(-1)^{l}(-1)^{k} \int_{-\infty}^{\infty} U_{n+r+s-l-1}^{(r+s+k-1)}(x) e^{-x^{2}} d x \\
& =\frac{1}{2^{k} k ! \sqrt{\pi}(r+s-1) ! 2^{r+s-1}} \sum_{l=0}^{n-k}\left(\begin{array}{l}
s \\
l
\end{array}\right)(-1)^{l} \\
& \times \frac{\left[\frac{n-k-l}{2}\right]}{\sum_{m=0}^{2}}(-1)^{m}\left(\begin{array}{c}
n+r+s-l-m-1 \\
m^{n}
\end{array}\right)(n+r+s-l-2 m-1)_{r+s+k-1} \\
& \times 2^{n+r+s-l-2 m-1} \int_{-\infty}^{\infty} x^{n-k-l-2 m} e^{-x^{2}} d x .
\end{aligned}
$$

Here, we note from (a) of Proposition 2 that

$$
\int_{-\infty}^{\infty} x^{n-k-l-2 m} e^{-x^{2}} d x=\left\{\begin{array}{cl}
0, & \text { if } n \neq k-l(\bmod 2), \\
\frac{(n-k-l-2 m) ! \sqrt{\pi}}{\left(\frac{n-k-l}{2}-m\right) ! 2^{n-k-l-2 m}}, & \text { if } n \equiv k-l(\bmod 2) .
\end{array}\right.
$$

From (53)-(55) and after some simplifications, we get

$$
\begin{aligned}
& \alpha_{n, r, s}(x)=\frac{1}{(r+s-1) !} \sum_{\substack { k=0 \\
\begin{subarray}{c}{l \equiv n-k(\bmod 2) \\
0 \leq l \leq n-k{ k = 0 \\
\begin{subarray} { c } { l \equiv n - k ( \operatorname { m o d } 2 ) \\
0 \leq l \leq n - k } }\end{subarray}}^{n} \sum_{m=0}^{\left[\frac{n-k-l}{2}\right]} \frac{\left(\begin{array}{l}
s \\
l
\end{array}\right)(-1)^{l}(-1)^{m}(n+r+s-l-m-1) !}{k ! m !\left(\frac{n-k-l}{2}-m\right) !} H_{k}(x) \\
& =\frac{1}{(r+s-1) !} \sum_{k=0}^{n} \sum_{j=0}^{\left[\frac{n-k}{2}\right]} \frac{(-1)^{n-k}\left(\begin{array}{c}
s \\
n-k-2 j
\end{array}\right)}{k !} \sum_{m=0}^{j} \frac{(-1)^{m}(r+s+k+2 j-m-1) !}{m !(j-m) !} H_{k}(x) \\
& =\frac{1}{(r+s-1) !} \sum_{k=0}^{n} \sum_{j=0}^{\left[\frac{n-k}{2}\right]} \frac{(-1)^{n-k}\left(\begin{array}{c}
s \\
n-k-2 j
\end{array}\right)(r+s+k+2 j-1) !}{k ! j !} \sum_{m=0}^{j} \frac{(-1)^{m}\langle-j\rangle_{m}}{m !\langle 1-r-s-k-2 j\rangle_{m}} H_{k}(x) \\
& =\sum_{k=0}^{n} \frac{(-1)^{n-k}}{(r+s-1) ! k !} \sum_{j=0}^{\left[\frac{n-k}{2}\right]} \frac{\left(\begin{array}{c}
s \\
n-k-2 j
\end{array}\right)(r+s+k+2 j-1) !}{j !}{ }_{1} F_{1}(-j ; 1-r-s-k-2 j ;-1) H_{k}(x) . .
\end{aligned}
$$

This shows (31) of Theorem 1.

Next, we let

$$
\alpha_{n, r, s}(x)=\sum_{k=0}^{n} C_{k, 2} L_{k}^{\alpha}(x) .
$$

Then, from (b) of Proposition 1, (48), and (52), integration by parts $k$ times and proceeding just as in (54), we obtain

$$
\begin{aligned}
C_{k, 2}= & \frac{1}{\Gamma(\alpha+k+1)(r+s-1) ! 2^{r+s-1}} \sum_{l=0}^{n-k}\left(\begin{array}{l}
s \\
l
\end{array}\right)(-1)^{l}(-1)^{k} \\
& \times \sum_{m=0}^{\left[\frac{n-k-l}{2}\right]}(-1)^{m}\left(\begin{array}{c}
n+r+s-l-m-1 \\
m
\end{array}\right)(n+r+s-l-2 m-1)_{r+s+k-1} \\
& \times 2^{n+r+s-l-2 m-1} \Gamma(n+\alpha-l-2 m+1) .
\end{aligned}
$$


From (57) and (58) and after some simplifications, we have

$$
\begin{aligned}
\alpha_{n, r, s}(x)= & \sum_{k=0}^{n} \frac{2^{n}(-1)^{n-k} L_{n-k}^{\alpha}(x)}{(r+s-1) ! \Gamma(\alpha+n-k+1)} \sum_{m=0}^{\left[\frac{k}{2}\right]} \frac{\left(-\frac{1}{4}\right)^{m}}{m !} \\
& \times \sum_{l=0}^{k-2 m} \frac{\left(\begin{array}{l}
s \\
l
\end{array}\right)\left(-\frac{1}{2}\right)^{l}(n+r+s-l-m-1) ! \Gamma(n+\alpha-l-2 m+1)}{(k-l-2 m) !} .
\end{aligned}
$$

Here, the innermost sum of (59) is equal to

$$
\begin{gathered}
\frac{(n+r+s-m-1) ! \Gamma(n+\alpha-2 m+1)}{(k-2 m) !} \sum_{l=0}^{k-2 m} \frac{\left(-\frac{1}{2}\right)^{l}\langle 2 m-k\rangle_{l}\langle-s\rangle_{l}}{l !\langle m+1-n-r-s\rangle_{l}\langle 2 m-n-\alpha\rangle_{l}} \\
=\frac{(n+r+s-m-1) ! \Gamma(n+\alpha-2 m+1)}{(k-2 m) !}{ }_{2} F_{2}\left(2 m-k,-s ; m+1-n-r-s, 2 m-n-\alpha ;-\frac{1}{2}\right) .
\end{gathered}
$$

Combining (59) and (60), we get

$$
\begin{aligned}
\alpha_{n r, s}(x)= & \sum_{k=0}^{n} \frac{2^{n}(-1)^{n-k}}{(r+s-1) ! \Gamma(\alpha+n-k+1)} \sum_{m=0}^{\left[\frac{k}{2}\right]} \frac{\left(-\frac{1}{4}\right)^{m}(n+r+s-m-1) ! \Gamma(n+\alpha-2 m+1)}{m !(k-2 m) !} \\
& \times{ }_{2} F_{2}\left(2 m-k,-s ; m+1-n-r-s, 2 m-n-\alpha ;-\frac{1}{2}\right) L_{n-k}^{\alpha}(x) .
\end{aligned}
$$

This completes the proof for (32) of Theorem 1.

Let us put

$$
\alpha_{n, r, S}(x)=\sum_{k=0}^{n} C_{k, 3} P_{k}(x) .
$$

From (c) of Proposition 1, (48), and (52), integration by parts $k$ times, (b) of Proposition 2 and after some simplifications, we obtain

$$
\begin{aligned}
& C_{k, 3}=\sum_{\substack{0 \leq l \leq n-k \\
l \equiv n-k(\bmod 2)}} \sum_{m=0}^{\left[\frac{n-k-l}{2}\right]} \frac{(2 k+1)\left(\begin{array}{l}
s \\
l
\end{array}\right)(-1)^{l}(-1)^{m}(n+r+s-l-m-1) !\left(\frac{n+k-l}{2}-m+1\right) ! 2^{n+k-l-2 m+1}}{(r+s-1) ! m !\left(\frac{n-k-l}{2}-m\right) !(n+k-l-2 m+2) !} \\
& =\sum_{j=0}^{\left[\frac{n-k}{2}\right]} \frac{(2 k+1)\left(\begin{array}{c}
s \\
n-k-2 j
\end{array}\right)(-1)^{n-k}(r+s+k+2 j-1) !}{(r+s-1) ! j !\left\langle\frac{1}{2}\right\rangle_{k+j+1}} \sum_{m=0}^{j} \frac{\langle-j\rangle_{m}\left\langle-k-j-\frac{1}{2}\right\rangle_{m}}{m !\langle 1-r-s-k-2 j\rangle_{m}} \\
& =\sum_{j=0}^{\left[\frac{n-k}{2}\right]} \frac{(2 k+1)\left(\begin{array}{c}
s \\
n-k-2 j
\end{array}\right)(-1)^{n-k}(r+s+k+2 j-1) ! 2^{k+j}}{(r+s-1) ! j !(2 k+2 j+1) ! !}{ }^{2} F_{1}\left(-j,-k-j-\frac{1}{2} ; 1-r-s-k-2 j ; 1\right) .
\end{aligned}
$$

Combining (62) and (63), we have

$$
\begin{aligned}
\alpha_{n, r, s}(x)= & \sum_{k=0}^{n} \frac{(-1)^{n-k}(2 k+1) 2^{k}}{(r+s-1) !} \sum_{j=0}^{\left[\frac{n-k}{2}\right]} \frac{2^{j}\left(\begin{array}{c}
s \\
n-k-2 j
\end{array}\right)(r+s+k+2 j-1) !}{j !(2 k+2 j+1) ! !} \\
& \times{ }_{2} F_{1}\left(-j,-k-j-\frac{1}{2} ; 1-r-s-k-2 j ; 1\right) P_{k}(x) .
\end{aligned}
$$

This shows (35) of Theorem 1

Let us set

$$
\alpha_{n, r, s}(x)=\sum_{k=0}^{n} C_{k, 4} C_{k}^{(\lambda)}(x)
$$


From (d) of Proposition 1, (48) and (52), integration by parts $k$ times, (c) of Proposition 2 and after some simplifications, we have

$$
\begin{aligned}
C_{k, 4} & =\sum_{\substack{0 \leq l \leq n-k \\
l \equiv n-k(\bmod 2)}} \sum_{m=0}^{\left[\frac{n-k-l}{2}\right]} \frac{(-1)^{l}(k+\lambda) \Gamma(\lambda)\left(\begin{array}{l}
s \\
l
\end{array}\right)}{\sqrt{\pi}(r+s-1) !} \\
& \times \frac{(-1)^{m}(n+r+s-l-m-1) ! 2^{n-k-l-2 m} \Gamma\left(\frac{n-k-l+1}{2}-m\right)}{m !(n-k-l-2 m) ! \Gamma\left(k+\lambda+\frac{n-k-l}{2}-m+1\right)} \\
& =\sum_{j=0}^{\left[\frac{n-k}{2}\right]} \frac{(-1)^{n-k}(k+\lambda) \Gamma(\lambda)\left(\begin{array}{c}
s \\
n-k-2 j
\end{array}\right)(r+s+k+2 j-1) !}{(r+s-1) ! \Gamma(k+\lambda+j+1) j !} \sum_{m=0}^{j} \frac{\langle-j\rangle_{m}\langle-k-\lambda-j\rangle_{m}}{m !\langle 1-r-s-k-2 j\rangle_{m}} \\
& =\sum_{j=0}^{\left[\frac{n-k}{2}\right]} \frac{(-1)^{n-k}(k+\lambda) \Gamma(\lambda)\left(\begin{array}{c}
s \\
n-k-2 j
\end{array}\right)(r+s+k+2 j-1) !}{(r+s-1) ! \Gamma(k+\lambda+j+1) j !} \\
& \times{ }_{2} F_{1}(-j,-k-\lambda-j ; 1-r-s-k-2 j ; 1) .
\end{aligned}
$$

Combining (64) and (65), we obtain

$$
\begin{aligned}
\alpha_{n, r, s}(x)= & \sum_{k=0}^{n} \frac{(-1)^{n-k} \Gamma(\lambda)(k+\lambda)}{(r+s-1) !} \sum_{j=0}^{\left[\frac{n-k}{2}\right]} \frac{\left.\begin{array}{c}
s \\
n-k-2 j
\end{array}\right)(r+s+k+2 j-1) !}{j ! \Gamma(k+\lambda+j+1)} \\
& \times{ }_{2} F_{1}(-j,-k-\lambda-j ; 1-r-s-k-2 j ; 1) C_{k}^{\lambda}(x) .
\end{aligned}
$$

This shows (36) of Theorem 1.

Let us let

$$
\alpha_{n, r, s}(x)=\sum_{k=0}^{n} C_{k, 5} P_{k}^{(\alpha, \beta)}(x) .
$$

From (e) of Proposition 1, (48) and (52), integration by parts $k$ times, (d) of Proposition 2 and after some simplifications, we get

$$
\begin{aligned}
C_{k, 5} & =\frac{(-1)^{k}(2 k+\alpha+\beta+1) \Gamma(k+\alpha+\beta+1)}{\Gamma(\alpha+k+1) \Gamma(\beta+k+1)(r+s-1) !} \sum_{l=0}^{n-k}\left(\begin{array}{l}
s \\
l
\end{array}\right)(-1)^{l}(-1)^{k} \\
& \times \sum_{m=0}^{\left.\frac{[n-k-l}{2}\right]}(-1)^{m}\left(\begin{array}{c}
n+r+s-l-m-1 \\
m
\end{array}\right)(n+r+s-l-2 m-1)_{r+s+k-1} 2^{n+k-l-2 m} \\
& \times \sum_{t=0}^{n-k-l-2 m}\left(\begin{array}{c}
n-k-l-2 m \\
t
\end{array}\right)(-1)^{n-k-l-t} 2^{t} \frac{\Gamma(k+\alpha+1) \Gamma(k+\beta+t+1)}{\Gamma(2 k+\alpha+\beta+t+2)} .
\end{aligned}
$$

It can be seen that the innermost sum of (67) is equal to

$$
\frac{(-1)^{n-k-l} \Gamma(k+\alpha+1) \Gamma(k+\beta+1)}{\Gamma(2 k+\alpha+\beta+2)}{ }_{2} F_{1}(k+l+2 m-n, k+\beta+1 ; 2 k+\alpha+\beta+2 ; 2) .
$$


By making use of (66)-(68), we finally obtain

$$
\begin{aligned}
\alpha_{n, r, s}(x)= & \sum_{k=0}^{n} \frac{(-2)^{n+k} \Gamma(k+\alpha+\beta+1)}{(r+s-1) ! \Gamma(2 k+\alpha+\beta+1)} \sum_{m=0}^{\left[\frac{n-k}{2}\right]} \frac{\left(-\frac{1}{4}\right)^{m}}{m !} \\
& \times \sum_{l=0}^{n-k-2 m} \frac{\left(\frac{1}{2}\right)^{l}\left(\begin{array}{l}
s \\
l
\end{array}\right)(n+r+s-l-m-1) !}{(n-k-l-2 m) !} \\
& \times{ }_{2} F_{1}(k+l+2 m-n, k+\beta+1 ; 2 k+\alpha+\beta+2 ; 2) P_{k}^{(\alpha, \beta)}(x) .
\end{aligned}
$$

This finishes up the proof for (35) of Theorem 1.

For Theorem 2, we only need to observe the following. From (48) and (50), we see that the only difference between $\alpha_{n, r, s}(x)$ and $\beta_{n, r, s}(x)$ (see (4), (5)) are the alternating sign $(-1)^{l}$ in their sums. These result in the differences $(-1)^{n-k}$, for (33) and (38), (35) and (40), (36) and (41), the difference ${ }_{2} F_{2}$, for (34) and (39), and the difference $(-1)^{l}$, for (37) and (42).

\section{Proof of Theorem 3}

Here, we will show only (45) and (47) in Theorem 3, while leaving (43), (44) and (46) as exercises to the reader.

Lemma 3. Let $n, r, s$ be nonnegative integers with $r+s \geq 1$. The following identity holds:

$$
\begin{gathered}
\sum_{i_{1}+\cdots+i_{r}+j_{1}+\cdots+j_{s}} V_{i_{1}}(x) \cdots V_{i_{r}}(x) W_{j_{1}}(x) \cdots W_{j_{s}}(x) \\
=\frac{1}{(r+s-1) ! 2^{r+s-1}} \sum_{i=0}^{n} \sum_{j=0}^{i}(-1)^{j}\left(\begin{array}{c}
r \\
j
\end{array}\right)\left(\begin{array}{c}
s \\
i-j
\end{array}\right) U_{n+r+s-i-1}^{(r+s-1)}(x) \\
=\frac{1}{(r+s-1) ! 2^{r+s-1}} \sum_{i=0}^{n}\left(\begin{array}{c}
s \\
i
\end{array}\right){ }_{2} F_{1}(-i,-r ; s-i+1 ;-1) U_{n+r+s-i-1}^{(r+s-1)}(x) .
\end{gathered}
$$

Proof. The identity in (69) is stated in [18] and can be deduced from (16) and (17). On the other hand, the identity in (70) follows from the elementary observation

$$
\sum_{j=0}^{i}(-1)^{j}\left(\begin{array}{l}
r \\
j
\end{array}\right)\left(\begin{array}{c}
s \\
i-j
\end{array}\right)=\left(\begin{array}{l}
s \\
i
\end{array}\right){ }_{2} F_{1}(-i,-r ; s-i+1 ;-1) .
$$

With $\gamma_{n, r, s}(x)$ as in (6), we put

$$
\gamma_{n, r, s}(x)=\sum_{k=0}^{n} C_{k, 3} P_{k}(x)
$$


Then, from (c) of Proposition 1, (70) and (52), (b) of Proposition 2, integration by parts $k$ times and after some simplifications, we have

$$
\begin{aligned}
C_{k, 3}= & \sum_{\substack{0 \leq i \leq n-k \\
i \equiv n-k(\bmod 2)}} \sum_{m=0}^{\left[\frac{n-k-i}{2}\right]} \frac{(2 k+1)\left(\begin{array}{l}
s \\
i
\end{array}\right)_{2} F_{1}(-i,-r ; s-i+1 ;-1)}{(r+s-1) ! m !} \\
\times & \frac{(-1)^{m}(n+r+s-i-m-1) ! 2^{n+k-i-2 m+1}\left(\frac{n+k-i}{2}-m+1\right) !}{\left(\frac{n-k-i}{2}-m\right) !(n+k-i-2 m+2) !} \\
= & \sum_{l=0}^{\left[\frac{n-k}{2}\right]} \sum_{m=0}^{l} \frac{(2 k+1)\left(\begin{array}{c}
s \\
n-k-2 l
\end{array}\right) 2 F_{1}(k+2 l-n,-r ; s+k+2 l-n+1 ;-1)}{(r+s-1) !} \\
& \times \frac{2^{2 k+2 l+1}\left(-\frac{1}{4}\right)^{m}(r+s+k+2 l-m-1) !(k+l-m+1) !}{m !(l-m) !(2 k+2 l-2 m+2) !} .
\end{aligned}
$$

By making use of (10), we see that (72) is equal to

$$
\begin{aligned}
C_{k, 3} & =\sum_{l=0}^{\left[\frac{n-k}{2}\right]} \frac{(2 k+1)\left(\begin{array}{c}
s \\
n-k-2 l
\end{array}\right){ }_{2} F_{1}(k+2 l-n,-r ; s+k+2 l-n+1 ;-1)}{(r+s-1) !} \\
& \times \frac{(r+s+k+2 l-1) !}{l ! 2\left\langle\frac{1}{2}\right\rangle_{k+l+1}} \sum_{m=0}^{l} \frac{\langle-l\rangle_{m}\left\langle-k-l-\frac{1}{2}\right\rangle_{m}}{m !\langle 1-r-s-k-2 l\rangle_{l}} \\
& =\sum_{l=0}^{\left[\frac{n-k}{2}\right]} \frac{(2 k+1) 2^{k+l}(r+s+k+2 l-1) !\left(\begin{array}{c}
s \\
n-k-2 l
\end{array}\right)}{(r+s-1) !(2 k+2 l+1) ! ! l !} \\
& \times{ }_{2} F_{1}\left(-l,-k-l-\frac{1}{2} ; 1-r-s-k-2 l ; 1\right) \\
& \times{ }_{2} F_{1}(k+2 l-n,-r ; s+k+2 l-n+1 ;-1) .
\end{aligned}
$$

Combining (71) and (73), we obtain

$$
\begin{aligned}
\gamma_{n, r, s}(x)= & \sum_{k=0}^{n} \frac{(2 k+1) 2^{k}}{(r+s-1) !} \sum_{l=0}^{\left.\frac{n-k}{2}\right]} \frac{2^{l}(r+s+k+2 l-1) !\left(\begin{array}{c}
s \\
n-k-2 l
\end{array}\right)}{(2 k+2 l+1) ! ! l !} \\
& \times{ }_{2} F_{1}\left(-l,-k-l-\frac{1}{2} ; 1-r-s-k-2 l ; 1\right) \\
& \times{ }_{2} F_{1}(k+2 l-n,-r ; s+k+2 l-n+1 ;-1) P_{k}(x) .
\end{aligned}
$$

This shows (45) of Theorem 3.

Lastly, we let

$$
\gamma_{n, r, s}(x)=\sum_{k=0}^{n} C_{k, 5} P_{k}^{(\alpha, \beta)}(x)
$$

Then, from (e) of Proposition 1, (70) and (52), (d) of Proposition 2, integration by parts $k$ times and after some simplifications, we obtain

$$
\begin{aligned}
C_{k, 5}= & \frac{(-2)^{n+k} \Gamma(k+\alpha+\beta+1)}{(r+s-1) ! \Gamma(2 k+\alpha+\beta+1)} \sum_{i=0}^{n-k}\left(-\frac{1}{2}\right)^{i}\left(\begin{array}{l}
s \\
i
\end{array}\right){ }_{2} F_{1}(-i,-r ; s-i+1 ;-1) \\
& \times \sum_{m=0}^{\left[\frac{n-k-i}{2}\right]} \frac{\left(-\frac{1}{4}\right)^{m}(n+r+s-i-m-1) !}{m !(n-k-i-2 m) !} \sum_{t=0}^{n-k-i-2 m} \frac{2^{t}\langle k+i+2 m-n\rangle_{t}\langle k+\beta+1\rangle_{t}}{t !\langle 2 k+\alpha+\beta+2\rangle_{t}} .
\end{aligned}
$$


Combining (74) and (75), we get

$$
\begin{aligned}
\gamma_{n, r, s}(x)= & \sum_{k=0}^{n} \frac{(-2)^{n+k} \Gamma(k+\alpha+\beta+1)}{(r+s-1) ! \Gamma(2 k+\alpha+\beta+1)} \sum_{i=0}^{n-k}\left(-\frac{1}{2}\right)^{i}\left(\begin{array}{l}
s \\
i
\end{array}\right){ }_{2} F_{1}(-i,-r ; s-i+1 ;-1) \\
& \times \sum_{m=0}^{\left[\frac{n-k-i}{2}\right]} \frac{\left(-\frac{1}{4}\right)^{m}(n+r+s-i-m-1) !}{m !(n-k-i-2 m) !} \\
& \times{ }_{2} F_{1}(k+i+2 m-n, k+\beta+1 ; 2 k+\alpha+\beta+2 ; 2) P_{n}^{(\alpha, \beta)}(x) .
\end{aligned}
$$

This completes the proof for (45) of Theorem 3.

\section{Further Remarks}

Here, we note that (36) and (41) can be simplified further by making use of Gauss summation formula when $r+s<\lambda+1$. For this, let us recall the Gauss summation formula:

$$
{ }_{2} F_{1}(a, b ; c ; 1)=\frac{\Gamma(c) \Gamma(c-a-b)}{\Gamma(c-a) \Gamma(c-b)}, \quad(\operatorname{Re}(c-a-b)>0) .
$$

We also need the following formulas:

$$
\begin{gathered}
(n+2 j) !=2^{2 j} n !\left\langle\frac{n+1}{2}\right\rangle_{j}\left\langle\frac{n+2}{2}\right\rangle_{j},(n, j \geq 0), \\
(n-2 j) !=\frac{n !}{2^{2 j}\left\langle\frac{-n}{2}\right\rangle_{j}\left\langle\frac{1-n}{2}\right\rangle_{j}}, \quad(n \geq 2 j \geq 0) .
\end{gathered}
$$

We also observe the following:

$$
\begin{gathered}
\Gamma(k+\lambda+j+1)=\langle k+\lambda+1\rangle_{j}(k+\lambda)\langle\lambda\rangle_{k} \Gamma(\lambda), \\
\frac{\Gamma(\lambda+1-r-s)}{\Gamma(\lambda+1-r-s-j)}=(-1)^{j}\langle r+s-\lambda\rangle_{j^{\prime}} \\
\frac{\Gamma(1-r-s-k-2 j)}{\Gamma(1-r-s-k-j)}=\frac{(-1)^{j}(r+s+k+j-1) !}{(r+s+k+2 j-1) !} .
\end{gathered}
$$

Assume now that $r+s<\lambda+1$. Then, from (76), we have

$$
\begin{aligned}
& \frac{(-1)^{n-k} \Gamma(\lambda)(k+\lambda)}{(r+s-1) !} \sum_{j=0}^{\left[\frac{n-k}{2}\right]} \frac{\left(\begin{array}{c}
s \\
n-k-2 j
\end{array}\right)(r+s+k+2 j-1) !}{j ! \Gamma(k+\lambda+j+1)} \\
& \quad \times{ }_{2} F_{1}(-j,-k-\lambda-j ; 1-r-s-k-2 j ; 1) \\
& =\frac{(-1)^{n-k} \Gamma(\lambda)(k+\lambda)}{(r+s-1) !} \sum_{j=0}^{\left[\frac{n-k}{2}\right]} \frac{\left(\begin{array}{c}
s \\
n-k-2 j)
\end{array}\right)(r+s+k+2 j-1) !}{j ! \Gamma(k+\lambda+j+1)} \\
& \times \frac{\Gamma(1-r-s-k-2 j) \Gamma(\lambda+1-r-s)}{\Gamma(1-r-s-k-j) \Gamma(\lambda+1-r-s-j)} .
\end{aligned}
$$


By making use of (77)-(81), we see that (82) is equal to

$$
\begin{aligned}
& \frac{(-1)^{n-k}\left(\begin{array}{c}
s \\
n-k
\end{array}\right)\langle r+s\rangle_{k}}{\langle\lambda\rangle_{k}} \sum_{j=0}^{\left[\frac{n-k}{2}\right]} \frac{\left\langle\frac{k-n}{2}\right\rangle_{j}\left\langle\frac{k-n+1}{2}\right\rangle_{j}\langle r+s+k\rangle_{j}\langle r+s-\lambda\rangle_{j}}{j !\left\langle\frac{s-n+k+1}{2}\right\rangle_{j}\left\langle\frac{s-n+k+2}{2}\right\rangle_{j}\langle k+\lambda+1\rangle_{j}} \\
& =(-1)^{n-k}\left(\begin{array}{c}
s \\
n-k
\end{array}\right)\langle r+s\rangle_{k}\langle\lambda\rangle_{k}^{-1} \\
& \quad \times{ }_{4} F_{3}\left(\frac{k-n}{2}, \frac{k-n+1}{2}, r+s+k, r+s-\lambda ; \frac{s-n+k+1}{2}, \frac{s-n+k+2}{2}, k+\lambda+1 ; 1\right) .
\end{aligned}
$$

Now, from (83), we obtain the following alternative expressions for (36) and (41).

Theorem 4. Let $n, r, s$ be nonnegetive integers satisfying $1 \leq r+s<\lambda+1$. Then, we have the following alternative expressions for (36) and (41):

$$
\begin{aligned}
& \quad \sum_{i_{1}+\cdots+i_{r}+j_{1}+\cdots+j_{s}=n} U_{i_{1}}(x) \cdots U_{i_{r}}(x) V_{j_{1}}(x) \cdots V_{j_{s}}(x) \\
& =\sum_{k=0}^{n}(-1)^{n-k}\left(\begin{array}{c}
s \\
n-k
\end{array}\right)\langle r+s\rangle_{k}\langle\lambda\rangle_{k}^{-1} \\
& \quad \times{ }_{4} F_{3}\left(\frac{k-n}{2}, \frac{k-n+1}{2}, r+s+k, r+s-\lambda ; \frac{s-n+k+1}{2}, \frac{s-n+k+2}{2}, k+\lambda+1 ; 1\right) C_{k}^{(\lambda)}(x), \\
& i_{1}+\cdots+i_{r}+j_{1}+\cdots+j_{s}=n \\
& =\sum_{k=0}^{n}\left(\begin{array}{c}
s \\
n-k
\end{array}\right)\langle r+s\rangle_{k}\langle\lambda\rangle_{k}^{-1}(x) \cdots U_{i_{r}}(x) W_{j_{1}}(x) \cdots W_{j_{s}}(x) \\
& \quad \times{ }_{4} F_{3}\left(\frac{k-n}{2}, \frac{k-n+1}{2}, r+s+k, r+s-\lambda ; \frac{s-n+k+1}{2}, \frac{s-n+k+2}{2}, k+\lambda+1 ; 1\right) C_{k}^{(\lambda)}(x) .
\end{aligned}
$$

\section{Conclusions}

Let $n, r, s$ be nonnegative integers with $r+s \geq 1$. Then, we considered sums of finite products of Chebyshev polynomials of two different kinds, namely those of the second and third kind Chebyshev polynomials $\alpha_{n, r, s}(x)$ in (4), those of the second and fourth kind Chebyshev polynomials $\beta_{n, r, s}(x)$ in (5), and those of the third and fourth kind Chebyshev polynomials $\gamma_{n, r, s}(x)$ in (6).

As one motivation of the present research, we noticed that this problem can be viewed as a generalization of the classical linearization problem. As another motivation, we explained that the standard linearlization problem for Bernoulli polynomials yields the identity (47) which in turn gives a variant of Miki's identity and the famous Faber-Pandharipande-Zagier identity.

In a related paper [18], we represented each of $\alpha_{n, r, s}(x), \beta_{n, r, s}(x)$, and $\gamma_{n, r, s}(x)$ as linear combinations of all kinds of Chebyshev polynomials. Here, as a continuation of this work, we expressed each of them as linear combinations of the orthogonal polynomials $H_{n}(x), L_{n}^{\alpha}(x), P_{n}(x), C_{n}^{(\lambda)}(x)$, and $P_{n}^{(\alpha, \beta)}(x)$. We saw that the coefficients involve some terminating hypergeometric functions ${ }_{2} F_{1},{ }_{1} F_{1},{ }_{2} F_{2}$, and ${ }_{4} F_{3}$. These were done by explicit computations.

We emphasize that, whereas all the results so far, except for [18], treated sums of finite products of some polynomials of a single kind, this paper considered those of two different kinds. We would like to continue to study this line of research, as our immediate future projects. Indeed, the cases of sums of finite products of Chebyshev polynomials of the first kind and those of some other kind have not been treated here, as they are of a somewhat different nature. The results on them will appear elsewhere. Finally, it is very likely that the problems of expressing sums of finite products of 
orthogonal polynomials in terms of other orthogonal polynomials can be generalized to the cases of $q$-orthogonal polynomials.

Author Contributions: T.K. and D.S.K. conceived of the framework and structured the whole paper; D.S.K. and T.K. wrote the paper; J.K. paid for the article processing charge of the paper; H.L. typed the paper; D.S.K. and T.K. completed the revision of the article. All authors have read and agreed to the published version of the manuscript.

Funding: This work was supported by the National Research Foundation of Korea (NRF) grant funded by the Korea government (MEST) (No. 2017R1E1A1A03070882).

Conflicts of Interest: The authors declare no conflicts of interest.

\section{References}

1. Sánchez-Ruiz, J.; Dehesa, J.S. Some connection and linearization problems for polynomials in and beyond the Askey scheme. J. Comput. Appl. Math. 2001, 133, 579-591. [CrossRef]

2. Kim, T.; Kim, D.S.; Jang, L.C.; Jang, G.-W. Sums of finite products of Genocchi functions. Adv. Differ. Equ. 2017, 2017, 268. [CrossRef]

3. Faber, C.; Pandharipande, R. Hodge Integrals and Gromov-Witten Theory. Invent. Math. 2000, 139, 173-199.

4. Dunne, G.V.; Schubert, C. Bernoulli Number Identities from Quantum Field Theory and Topological String Theory. Commun. Number Theory Phys. 2013, 7, 225-249. [CrossRef]

5. Gessel, I.M. On Miki's identity for Bernoulli numbers. J. Number Theory 2005, 110, 75-82. [CrossRef]

6. Miki, H. A relation between Bernoulli numbers. J. Number Theory 1978, 10, 297-302. [CrossRef]

7. Shiratani, K.; Yokoyama, S. An application of p-adic convolutions. Mem. Fac. Sci. Kyushu Univ. Ser. Math. 1982, 36, 73-83. [CrossRef]

8. Kim, D.S.; Dolgy, D.V.; Kim, T.; Rim, S.-H. Identities involving Bernoulli and Euler polynomials arising from Chebyshev polynomials. Proc. Jangjeon Math. Soc. 2012, 15, 361-370.

9. Kim, D.S.; Kim, T.; Lee, S.-H. Some identities for Bernoulli polynomials involving Chebyshev polynomials. J. Comput. Anal. Appl. 2014, 16, 172-180.

10. Kim, D.S.; Kim, T.; Rim, S.-H. Some identities involving Gegenbauer polynomials. Adv. Differ. Equ. 2012, 219, 11. [CrossRef]

11. Kim, D.S.; Kim, T.; Rim, S.-H.; Lee, S.H. Hermite polynomials and their applications associated with Bernoulli and Euler numbers. Discret. Dyn. Nat. Soc. 2012, 2012, 974632. [CrossRef]

12. Kim, D.S.; Rim, S.-H.; Kim, T. Some identities on Bernoulli and Euler polynomials arising from orthogonality of Legendre polynomials. J. Inequal. Appl. 2012, 227, 8. [CrossRef]

13. Kim, T.; Kim, D.S. Extended Laguerre polynomials associated with Hermite, Bernoulli, and Euler numbers and polynomials. Abstr. Appl. Anal. 2012, 2012, 957350. [CrossRef]

14. Kim, T.; Kim, D.S.; Dogy, D.V. Some identities on Bernoulli and Hermite polynomials associated with Jacobi polynomials. Discret. Dyn. Nat. Soc. 2012, 2012, 584643. [CrossRef]

15. Andrews, G.E.; Askey, R.; Roy, R. Special Functions, in Encylopedia of Mathematics and Its Applications 71; Cambridge University Press: Cambridge, UK, 1999.

16. Beals, R.; Wong, R. Special Functions and Orthogonal Polynomials; Cambridge Studies in Advanced Mathematics 153; Cambridge University Press: Cambridge, UK, 2016.

17. Mason, J.C.; Handscomb, D.C. Chebyshev Polynomials; CHAPMAN \& Hall/CRC: Boca Raton, FL, USA, 2003.

18. Kim, T.; Dolgy, D.V.; Kim, D.S. Representing sums of finite products of Chebyshev polynomials of the second kind and Fibonacci polynomials in terms of Chebyshev polynomials. Adv. Stud. Contemp. Math. (Kyungshang) 2018, 28, 321-335.

19. Kim, T.; Kim, D.S.; Dology, D.V.; Kim, D. Representation by several orthogonal polynomials for sums of finite products of Chebysehv polynomials of the first, third and fourth kinds. Adv. Differ. Equ. 2019, 2019, 110. [CrossRef]

20. Kim, T.; Kim, D.S.; Kwon, J.; Dolgy, D.V. Expressing sums of finite products of Chebyshev polynomials of the second kind and of Fibonacci polynomials by several orthogonal polynomials. Mathematics 2018, 10, 210. [CrossRef]

(C) 2020 by the authors. Licensee MDPI, Basel, Switzerland. This article is an open access article distributed under the terms and conditions of the Creative Commons Attribution (CC BY) license (http://creativecommons.org/licenses/by/4.0/). 